\title{
sciendo
}

LanCog Lectures in Metaphysics 2013

\section{Modes of Being and Quantification ${ }^{1}$}

\author{
Peter van Inwagen \\ The University of Notre Dame
}

DOI: $10.2478 /$ disp-2014-0001

BIBLID [0873-626X (2014) 38; pp. 1-24]

If Pegasus existed, he would indeed be in space and time, but only because the word 'Pegasus' has spatio-temporal connotations, and not because 'exists' has spatio-temporal connotations. If spatio-temporal reference is lacking when we affirm the existence of the cube root of 27 , that is simply because a cube root is not a spatio-temporal kind of thing.

W. V. Quine, "On What There Is"

Many philosophers have held that being comes in various kinds or sorts or "modes". One thinks of Meinong's Existenz and Bestand, of Russell's existence and subsistence, of Sartre's être-en-soi and êtrepour-soi, and of Heidegger's Vorhandenheit, Zuhandenheit, and Existenz. But if there is more than one mode of being, what is the relation between the several modes of being and the existential quantifier? Does not the fact that there is a single existential quantifier strongly suggest that there is a single mode of being — or, perhaps better, that the concept "mode" has no application to being? I will put the idea that underlies this question in the form of an argument for the conclusion that there cannot be two or more modes of being.

Suppose we add to the language of first-order logic with identity a property abstraction operator, ' $\Pi$ '. This operator takes a variable and a sentence and makes a term in which that variable is not free. If the abstraction operator is applied to a variable $\alpha$ and a sentence in which $\alpha$ alone is free, the result is a closed term that denotes the property or attribute or quality expressed by that open sentence: the expression ' $\Pi_{x} x$ is wise' ("The property of being an $x$ such that $x$ is wise") denotes wisdom, and ' $\Pi z z$ is brave' denotes bravery or cour-

${ }^{1}$ This paper is the text of the first of two LanCog Lectures in Metaphysics, which were presented at the University of Lisbon on 12 and 14 June, 2013.

Disputatio, Vol. VI, No. 38, May 2014

Received: 15/10/2013 Accepted: 15/10/2013 
age - and so on. It seems to me that my description of this operator is unproblematical - provided at least that we do not insist that the terms it forms always have referents. ${ }^{2}$ (One would not want to suppose that there was such a property as $\Pi_{x} x$ is a property and it is not the case that $x$ has $x$.) I will suppose, however, that any such term that is formed from a meaningful and unambiguous open sentence denotes a property unless that term's denoting a property implies a contradiction. ${ }^{3}$ That may not be a very satisfactory "comprehension principle", but, really, no one knows what to do with Russell's Paradox.

Now consider the property $\prod_{x} \exists_{y} y=x$ - the property of being an $x$ is such that there is or exists a $y$ such that $y$ is identical with $x$, or the property of being a thing such that there is or exists something that is that thing. ${ }^{4}$ If we use the word 'being' as a noun or substantive, and as a mass term rather than a count-noun — as the English equivalent of Sein or être (mass term, not count noun) - then (surely?) what this word denotes is nothing other than that property. At any rate, I don't see what else its referent could be — just as I don't see the referent of 'wisdom' could be if not $\Pi_{x} x$ is wise. And if that is so, then there cannot be multiple modes of being, owing simply to the fact that open sentences like ' $\exists y y=x$ ' and 'something is $x$ ' express — if Kant and Frege will forgive me — a single, perfectly determinate property, a property had by — if Meinong will forgive me - everything.

This argument seems to me to be unanswerable — provided that there is a unique existential quantifier. But suppose that what we have learned to call "the" existential quantifier is not unique. Suppose that there is indeed more than one mode of being, and that each

${ }^{2}$ And provided we recognize that quantification into such terms (e.g., ‘ $\exists x \exists y x$ has $\Pi_{z} y$ is larger than $z$ ') is quantification into an intensional context.

${ }^{3}$ This does not rule out such properties as $\Pi_{x} \neq x_{x}$ and $\Pi_{x} x$ is a cubical ball. There is no contradiction in the existence of such properties - the contradiction would be in their instantiation.

${ }^{4}$ It is, to say the least, implausible to suppose that the existence of this property entails a contradiction. For one thing, free logics aside, ' $\exists y y=x$ ' is equivalent to ' $x=x^{\prime}$, and the existence of the property self-identity can hardly be supposed to entail a contradiction. 
mode of being has, so to speak, its own existential quantifier.

It will be convenient, when we are supposing that there is more than one mode of being, also to suppose that we have an actual specification of these modes. I will suppose that there are exactly two. I will call them 'existenz' and 'subsistence'. (Note that the former word has the German spelling.) Existenz, I stipulate, is the mode of being of concrete objects, and subsistence is the mode of being of abstract objects (whatever that distinction may come to). Everything I say will apply, with only trivial modifications, to any "list" of the modes of being you may care to put forward — Sartre's or Heidegger's for example.

Suppose, then, that a philosopher who holds that these are the two modes of being, existenz and subsistence, replies to the above argument by contending that there is a primitive and irreducible quantificational apparatus specific to existenz and a primitive and irreducible quantificational apparatus specific to subsistence. This is precisely the position of a certain fictional philosopher I'll call "McHeidegger" — a personification of the interpretation of Heidegger's philosophy of being that Kris McDaniel has presented in his well-known essay, "Ways of Being." ${ }^{5}$ My examination of the relation between quantification and modes of being will take the form of a critique of McHeidegger's philosophy — the only philosophy I know of that addresses this issue. I will not consider the question whether Heidegger accepted all or any of the theses of the meta-ontology ${ }^{6}$ ascribed to him in McDaniel's essay. ${ }^{7}$ I shall be concerned, rather, with some questions about that meta-ontology itself, that meta-ontology considered as a philosophical position, whoever may have accepted it. (McDaniel himself either accepts it or comes very close to accept-

${ }^{5}$ Included in David Chalmers, David Manley, and Ryan Wasserman (eds.) Metametaphysics: New Essays on the Foundations of Ontology (Oxford: Oxford University Press, 2009), pp. 290-319).

${ }^{6}$ I use this word in the sense it has in current analytical metaphysics. It is not meant to be a translation of Heidegger's 'Metontologie'.

${ }^{7}$ And I will not be concerned with the point that existenz and subsistence do not even remotely resemble the modes of being that actually figure in Heidegger's Seinsphilosophie. (My "existenz" is of course not Heidegger's Existenz. My “existenz” is, however, very like Meinong's Existenz.) 
ing it.) These questions have primarily to do with the implications of the meta-ontology for the logical validity of inferences involving particularity and universality.

This was prologue. Now the play. (Some of the ideas sketched in the prologue will be re-stated in the play — but in considerably more detail.)

Let us suppose that, in addition to the familiar "generic" quantifiers (the particular quantifier ' ' $\exists$ ' and its dual, the universal quantifier " $\forall$ '9), there are two pairs of "specific" quantifiers ${ }^{10}$, each member of one of the pairs being the dual of the other. And let us suppose that the two pairs are:

The concrete quantifiers

E the existenzial quantifier

A the general quantifier
The abstract quantifiers

$\checkmark$ the subsistential quantifier

$\wedge$ the inclusive quantifier.

The motive for this supposition is to be found in the thesis that there are two modes of being and that each of them is in some sense so fundamental a feature of reality that it requires its own quantificational apparatus. The existenzial quantifier (we suppose) expresses existenz (the verb is 'exizt'), the mode of being enjoyed by concrete

${ }^{8}$ I refrain from calling this operator by its customary name in order to avoid begging any questions - and also to avoid giving it a name that might be confused with the name given to one of the specific quantifiers.

${ }^{9}$ I follow the usage of Donald Kalish and Richard Montague: the symbols ' $\exists$ ' and ' $\forall$ ' are called quantifiers and expressions like ' $\exists z$ ' and ' $\forall y$ ' are called quantifier phrases. (Other writers call ' $\exists z$ ' and ' $\forall y$ ' quantifiers.)

${ }^{10}$ McDaniel's terms are 'unrestricted quantifier' and 'restricted quantifier'. (He does, however, speak of the generic and specific senses of 'being' and does sometimes apply these terms to the quantifiers that "express" those senses.) He recognizes (303) that his terminology is not entirely satisfactory, since "there is a sense in which any semantically primitive quantifier is an unrestricted quantifier." 
particulars or reale Gegenstände (pick some term that you favor; a precise statement of the mode of being expressed by ' $E$ ' is not important for our purposes; what matters is that this operator should express one of two modes of being). And the subsistential quantifier (we further suppose) expresses subsistence or Bestand, the mode of being enjoyed by abstract objects or ideale Gegenstände (or what you will). So an existenzial quantification on a predicate is true just in the case that some "exiztent" satisfies that predicate, and a general quantification on a predicate is true just in the case that all exiztents satisfy that predicate. And so for the subsistential and inclusive quantifiers, mutatis mutandis. And, finally, the particular quantifier expresses "generic being" - the "common element" of existenz and subsistence; that commonality in virtue of which both modes of being are modes of being, and the domain of the universal quantifier comprises everything that participates in either mode of being.

According to McHeidegger, the specific quantifiers are the fundamental and semantically primitive quantifiers, and the generic quantifiers are disjunctive and derived. ${ }^{11}$ (I don't mean to imply that McDaniel supposes that existenz and subsistence are the modes of being recognized by the real philosopher named 'Martin Heidegger'.)

Let us say that a formal mixed inference is an inference or argument whose constituent sentences (that is, its premises and its conclusion) contain quantifiers drawn from at least two of the three pairs $\exists / \forall$, $\mathrm{E} / \mathrm{A}$, and $\mathrm{V} / \wedge$. And let us say that an informal mixed inference is an inference or argument expressed in natural language that those who

11 'Disjunctive' has to be understood with some care. McDaniel says (306), "Heidegger recognizes a generic sense of 'being' that . . . represents something akin to a mere disjunction of the metaphysically basic ways of being." (Italics in original.) But he had earlier (304) said, “ . . . Heidegger does not seem to think that the generic sense of 'being' is merely the disjunction of the various specific senses of 'being.'" I take it that the position McDaniel ascribes to Heidegger is something like this (assuming for the sake of the example that subsistence and existenz are the modes of being): 'exists-generic' does not mean 'either subsists or exizts'; but the meaning of 'exists-generic' is such that 'it exists-generic' can properly be said of things that subsist and can, with equal propriety, be said of things that exizt. 
affirm the reality of existenz and subsistence as distinct modes of being and who insist that each mode be supplied with "its own" pair of specific quantifiers would find it natural to represent formally as a formal mixed inference.

I begin by presenting an argument that certainly seems to be an informal mixed inference by the terms of this definition — owing to the fact that it involves quantification (the quantificational apparatus is informal: the quantificational apparatus of ordinary English) over both mathematicians and mathematical problems, and (given that there are such modes of being as existenz and subsistence) one must suppose that mathematicians exizt and mathematical problems subsist.

\section{The Informal Mixed Argument}

There is a problem in algebraic topology that I. M. James is unable to solve

\section{M. James is an algebraic topologist}

Every algebraic topologist is able to solve any problem in algebraic topology that any algebraic topologist is able to solve

If there is a problem in algebraic topology that no algebraic topologist is able to solve, then no mathematician is able to solve that problem

Every problem in algebraic topology is a mathematical problem hence,

There is a mathematical problem that no mathematician is able to solve.

And the Informal Argument certainly seems to be valid. After all, if it were presented as a "translation exercise" in a logic course, the instructor would certainly regard the following argument as a correct representation of the Informal Argument in (as Quine calls it), the canonical language of first-order logic: 
The Generic Formal Argument

$\exists x(x$ is a problem in algebraic topology \& $\sim$ I. M. James is able to solve $x$ )

I. M. James is an algebraic topologist

$\forall x \forall_{y} \forall z$ ( $x$ is an algebraic topologist \& $y$ is an algebraic topologist $\& z$ is a problem in algebraic topology $\cdot \rightarrow \cdot x$ is able to solve $z \leftrightarrow$ $y$ is able to solve $z$ )

$\forall x$ ( $x$ is a problem in algebraic topology $\& \sim \exists_{y}$ ( $y$ is an algebraic topologist $\& y$ is able to solve $x) \cdot \rightarrow \sim \exists_{y}(y$ is a mathematician $\& y$ is able to solve $x)$ )

$\forall x$ ( $x$ is a problem in algebraic topology $\rightarrow x$ is a mathematical problem)

hence,

$\exists x$ ( $x$ is a mathematical problem \& $\exists_{y}(y$ is a mathematician \& $y$ is able to solve $x)$ ).

And it would be easy to show that the Generic Formal Argument was valid by the methods set out in whatever the textbook in the course happened to be (the methods that one of my teachers liked to refer to collectively as "quantifier dropping and horseshoe pushing").

3

Suppose, however, that the only quantifiers we had at our disposal were the four specific quantifiers mentioned in section 1 - the concrete quantifiers and the abstract quantifiers. Then (owing to the distinct modes of being enjoyed by mathematicians, on the one hand, and mathematical problems, on the other) if we wished to translate the Informal Argument into the quantifier-variable idiom, we should have to replace each generic quantifier in the Generic Formal Argument with the appropriate specific quantifier — thus producing the following argument (a "formal mixed inference"): 
The Specific Formal Argument

$V_{x}(x$ is a problem in algebraic topology $\& \sim$ I. M. James is able to solve $x$ )

I. M. James is an algebraic topologist

$A_{x} A_{y} \wedge_{z}(x$ is an algebraic topologist $\& y$ is an algebraic topologist $\& z$ is a problem in algebraic topology $\cdot \rightarrow \cdot x$ is able to solve $z \leftrightarrow$ $y$ is able to solve $z$ )

$\Lambda_{x}\left(x\right.$ is a problem in algebraic topology $\& \sim \mathrm{E}_{y}$ ( $y$ is an algebraic topologist \& $y$ is able to solve $x) \cdot \rightarrow \sim \mathrm{E}_{y}(y$ is a mathematician \& $y$ is able to solve $x)$ )

$\Lambda_{x}(x$ is a problem in algebraic topology $\rightarrow x$ is a mathematical problem)

hence,

$V_{x}$ ( $x$ is a mathematical problem \& $\sim \mathrm{E}_{y}$ ( $y$ is a mathematician \& $y$ is able to solve $x)$ ).

And what rules shall we consult if we wish to determine whether this argument is valid? Or, more generally, what rules shall we consult if we wish to determine whether any given formal mixed inference is valid?

I will consider two ways to approach these questions. I'll call them the Deep Approach and the Shallow Approach.

\section{4}

Advocates of the Deep Approach will dispute my statement that the Specific Formal Argument is a correct formal representation of the Informal Argument. They will insist that all the terms (all the variables and all the singular terms) that appear in a correct formal representation of that argument must be "sorted". (Singular terms must be of two "sorts": terms of one sort denote exiztents and only exiztents and terms of the other denote subsistents and only subsistents. And variables, too, must be sorted: into a "sort" bound by and only 
by the concrete quantifiers and a sort bound by and only by the abstract quantifiers). Let us accede to their demand; let the "concrete" variables be ' $x$ ', ' $y$ ', ' $z$ ' . . . , and let the "abstract" variables be ' $x$ ', ' $y$ ', ' $z$ ' . . . And let us distinguish "concrete" and "abstract" singular terms by a similar notational device.

A correct formal representation of the Informal Argument should, as the Deep Approachers see matters, look something like this:

\section{The Specific Formal Argument (Sorted)}

$V \mathbf{X}(\mathrm{X}$ is a problem in algebraic topology $\& \sim \mathrm{I}$. M. James is able to solve $\mathbf{X})$

I. M. James is an algebraic topologist

$A_{x} A_{y} \wedge z(x$ is an algebraic topologist $\& y$ is an algebraic topologist $\& \mathrm{Z}$ is a problem in algebraic topology $\cdot \rightarrow \cdot x$ is able to solve $z \leftrightarrow y$ is able to solve $\mathbf{z}$ )

$\Lambda \mathrm{x}(\mathrm{X}$ is a problem in algebraic topology \& $\sim \mathrm{Ey}$ ( $\mathrm{y}$ is an algebraic topologist $\& y$ is able to solve $\mathrm{x}) \cdot \rightarrow \sim \mathrm{E}_{y}(\mathrm{y}$ is a mathematician $\& y$ is able to solve $\mathbf{X})$ )

$\mathrm{Ax}(\mathrm{X}$ is a problem in algebraic topology $\rightarrow \mathrm{x}$ is a mathematical problem)

hence,

$\mathrm{Vx}$ ( $\mathrm{X}$ is a mathematical problem \& $\sim \mathrm{E}_{y}$ ( $\mathrm{y}$ is a mathematician \& $y$ is able to solve $\mathbf{X})$ ).

Is this argument formally valid? The question is easily answered: it is. It is at any rate formally valid according to the treatments of "manysorted logic" that are available in the literature. ${ }^{12}$ And I have no doubt

\footnotetext{
${ }^{12}$ See Herbert B. Enderton A Mathematical Introduction to Logic, 2nd Edn (Harcourt/Academic Press, 2001), Section 4.3 (pp. 295-299), "Many-sorted Logic." (I am grateful to Hannes Leitgeb and Chris Menzel for calling my attention to Enderton's treatment of many-sorted logic.) Actually, despite my knowing reference to "the treatments of 'many-sorted logic' available in the literature," this is the only such treatment I know of. But if there are others, I am confident that
} 
that, if an informal mixed inference is intuitively equivalent to a specific formal argument with sorted variables, the informal inference will be intuitively logically valid if and only if the specific formal argument is formally valid.

Is the Deep Approach to the problem of mixed inferences therefore satisfactory? That will depend on whether the Deep Approachers recognize, in addition to the four specific quantifiers and the sorted variables they bind, the generic quantifiers and unsorted variables. If they do not recognize generic quantifiers and unsorted variables, they will have adopted a language of insufficient expressive power; that is, there will be things that can be said that they cannot say general statements that can be expressed in English (and presumably in any natural language with an appropriate lexicon) that cannot be said in a formal language without generic quantification..$^{13}$ The most important of these are certain "cross-modal generalizations" - generalizations over objects some of which exizt and some of which subsist. For example:

Everything either exizts or subsists

Another cross-modal generalization that it may be impossible to express without generic quantification is:

Nothing both exizts and subsists.

I say that it may be impossible to express this statement without generic quantification because one might try to express it like this:

$$
\sim \mathrm{E}_{X} \vee \mathrm{y}(\mathrm{x}=\mathrm{y}) \text {. }
$$

(Or reverse the order of the existenzial and the subsistential quantifier-phrases.) Whether this device "works", however, depends on

they are equivalent to Enderton's. As Enderton says (p. 295), “As might be expected, nothing is drastically different from the usual one-sorted situation. None of the results of this section are at all deep, and most of the proofs are omitted." The generalization of one-sorted logic that Enderton presents is so simple and natural that there doesn't seem to be any possibility of a rival, non-equivalent development of many-sorted logic (other than those that correspond to rival, non-equivalent "developments" of ordinary one-sorted logic: a many-sorted free logic, for example).

${ }^{13}$ As McDaniel says (297), "The generic concept of being is indispensable." 
whether the logical vocabulary of our hypothetical language without generic quantification contains a generic or "unsorted" identity-sign, an identity-sign an occurrence of which can be flanked by terms of different sorts. ${ }^{14}$ Enderton's many-sorted logic (see note 11) does not have such a sign (in his logic, each sort of term has its "own" identitysign), but I should think that it would be easy enough to add one. Models for many-sorted logic associate non-overlapping universes of discourse with terms of different sorts, and, therefore, the obvious adaptation of the usual model-theoretic treatment of ' $=$ ' will have the consequence that sentences formed by flanking the generic identity sign with singular terms of different sorts will "automatically" be false, and sentences like ' $z=y$ ' and ' $x=I$. M. James' will automatically be unsatisfiable. (But I speak under correction. I am no logician - and, as my colleague of beloved memory, the late Ralph McInerny, once said in a very similar context, that is no idle boast.)

However this may be, it is impossible to express the proposition that everything either exizts or subsists without the use of the universal quantifier. (And those who believe that existenz and subsistence are distinct modes of being will regard this as an important thesis - even those of them who think that it is false.)

McDaniel will, however, point out that McHeidegger is not unable to express such important metaphysical theses as "Everything either exizts or subsists" and "Nothing both exizts and subsists". He will remind us that McHeidegger affirms the existence (or perhaps we should say the subsistence) of the generic sense of 'exist' (albeit he does not regard it as fundamental or primitive) and thus rejects the thesis that the only quantifiers we have at our disposal are the specific quantifiers. If McHeidegger is right, we also have the generic quantifiers, the particular quantifier and the universal quantifier, "at our disposal". To revert to the case of subsistence and existenz, we may express the proposition that everything either subsists or exizts in either of the following ways:

${ }^{14}$ Note that the following truth is also inexpressible in a language without generic identity: I. M. James is not identical with the Kervaire Invariant 1 Problem. 


$$
\begin{aligned}
& \forall x\left(\vee_{y}={ }_{x} \cdot \vee E_{y y}={ }_{x}\right) \\
& \forall x\left(\vee y y={ }_{x} \cdot \vee E_{y y}={ }_{x}\right) .{ }^{15}
\end{aligned}
$$

(In the first if the specific quantifiers can bind unsorted variables; in the second if the specific quantifiers can bind only sorted variables. I will assume in the sequel that the concrete quantifiers bind only "existenz" variables and that the abstract quantifiers bind only "subsistence" variables.) Of course, both expressions require the generic identity-sign, but, as we have seen, there seems to be no obstacle to adding the generic identity-sign to a many-sorted language.

But more must be said to enable the McHeiddegerian to deal with the problem of mixed inferences. There is still the problem of the validity of those arguments whose constituent sentences contain both generic and specific quantifiers. This argument, for example:

$$
\forall x\left(\vee y y={ }_{x} \cdot \mathrm{VE}_{y} \mathrm{~V}_{\mathrm{x}}\right)
$$$$
\sim \exists_{x} \vee y(x=y)
$$

hence,

$$
\forall x \mathrm{E}_{y} \mathrm{y}={ }_{x} .
$$

(Everything is either subsistent or exiztent; Nothing is subsistent; hence, Everything is exiztent.) Or this argument:

$\exists x \exists y$ the mode of being of $x$ is not the mode of being of $y$

$\forall x \forall y$ (the mode of being of $x$ is not the mode of being of $y \rightarrow \cdot\left(E_{z}\right.$ $\left.\left.{ }_{x}={ }_{z} \cdot \& \vee_{z_{y}}=z\right) \vee\left(\mathrm{E}_{z} y={ }_{z} \cdot \& V_{z_{x}}=z\right)\right)$

hence,

$$
\exists x \mathrm{E}_{\mathrm{x}}=\mathrm{y} \cdot \& \exists_{\mathrm{x}} \vee_{\mathrm{y}}=\mathrm{y} .
$$

(There are things that participate in different modes of being; If two things participate in different modes of being, one of them is exiztent and the other is subsistent; hence, Something is exiztent and something is subsistent.)

Now there is an obvious and easy general solution to the problem

${ }^{15}$ Or as the perhaps harder-to-parse ' $\forall x\left(V x x={ }_{x} \cdot V E_{X} x=x\right)$ '. 
of determining the validity of formal mixed inferences - general in that it applies to all mixed arguments, both those that contain only specific quantifiers and those that contain both generic and specific quantifiers. (It will also apply to arguments that contain only concrete quantifiers and arguments that contain only abstract quantifiers.) At the end of section 3, I said, "There are two ways to approach these questions. I'll call them the Deep Approach and the Shallow Approach". The Shallow Approach and "the obvious and easy solution to the problem" are one and the same device — to wit, systematically to replace all the specific quantifiers in the inference we are testing for validity with appropriately restricted generic quantifiers ("for purposes of determining validity", as one might say). We might, for example, introduce a predicate ' $Z$ ' to express existenz, and a predicate ' $\mathbf{S}$ ' to express subsistence. (That is, ' $\mathbf{Z} x$ ' abbreviates ' $x$ exizts' and 'Sy' abbreviates ' $y$ subsists' and so on.) So, for example, replacing the specific quantifiers in the following two sentences with appropriately restricted generic quantifiers (binding generic variables):

$$
\forall x\left(\vee y y=x \cdot \mathrm{VE}_{y} y=x\right)
$$

$$
A x \wedge x \quad x \neq x
$$

yield, respectively,

$$
\forall x\left(\exists_{y}\left(\mathbf{Z}_{y} \&_{y}=x\right) \vee \exists_{y}\left(\mathbf{S}_{y} \&_{y}={ }_{x}\right)\right)
$$

and

$$
\forall x \forall_{y}\left(\mathbf{Z}_{x} \& \mathbf{S}_{y} \cdot \rightarrow_{x} \neq y\right) .
$$

Let us call the sentence obtained by so replacing all the specific quantifiers in a sentence with restricted generic quantifiers the "generic representation" of that sentence. And we may say that an inference or argument is the "generic representation" of a "specific" inference or argument (an inference or argument containing specific quantifiers) if it is the result of replacing each of its constituent sentences with its generic representation. We now consider 
The Generic-representation Criterion of Formal Validity (sc. of specific arguments)

A specific argument is formally valid if and only if its generic representation is formally valid (i.e., valid in ordinary quantifier logic).

(All formal mixed inferences are specific arguments, but arguments containing only concrete quantifiers and arguments containing only abstract quantifiers are specific arguments and are not formal mixed inferences.) The section of Enderton's A Mathematical Introduction to Logic (note 11) that is devoted to many-sorted logic has a sub-section called "Reduction to One-Sorted Logic". The results presented in that sub-section imply that a specific argument that contains no generic quantifiers is valid in many-sorted logic if and only if it is valid according to the Generic-representation Criterion. It obviously follows that the same holds for specific arguments that do contain generic quantifiers.

There is a sense in which the Generic-representation Criterion treats ' $\mathbf{Z}$ ' and ' $\mathbf{S}$ ' as "logically inert", since they are not mentioned in the rules that one would consult to determine whether the generic representation of a formal mixed inference was valid — that is, the inference rules of ordinary (i.e., generic) quantifier logic. One who is applying the Generic-representation Criterion will treat, e.g., ' $\mathbf{Z}_{x}$ ' and 'Sy' as "just two more open sentences", sentences that have no more logical significance than ' $x$ is a mathematician' and ' $y$ is a mathematical problem'.

We need not, in fact, have introduced ' $\mathbf{Z}$ ' and ' $\mathbf{S}$ ' by stipulating that ' $Z_{x}$ ' was to abbreviate ' $x$ exizts' and that ' $S_{y}$ ' was to abbreviate ' $y$ subsists' (and so on). We could instead have introduced, e.g., ' $\mathbf{Z}_{x}$ ' and ' $S_{y}$ ' as abbreviations for, respectively, ' $E_{\boldsymbol{X} x}=x$ ' and ' $\mathrm{X} \mathbf{x}=y$ '. On that reading of ' $Z$ ' and ' $\mathbf{S}$ ', the sentence

$$
\forall x\left(\exists y\left(\mathbf{Z}_{y} \&_{y}=x\right) \vee \exists y\left(\mathbf{S}_{y} \&_{y}={ }_{x}\right)\right)
$$

is an abbreviation of:

$$
\forall x\left(\exists y\left(\mathrm{E}_{y} \mathbf{y}=y \cdot \&_{y}={ }_{x}\right) \vee \exists_{y}\left(\vee \mathrm{y} \mathbf{y}={ }_{y} \cdot \&_{y}=x\right)\right) .
$$

On that understanding of ' $\mathbf{Z}$ ' and ' $\mathbf{S}$ ', those symbols can be regarded as devices for "isolating" the all the occurrences of the specific quan- 
tifiers in a specific argument inside "logically inert" predicates. And we could say that, e.g., the argument

$\forall x\left(\operatorname{E}_{x x}={ }_{x} \cdot \rightarrow(x\right.$ is a mathematician $\rightarrow \exists y(\vee x x=y \cdot \& y$ is a mathematical problem $\& \sim x$ is able to solve $y)$ ).

$\exists_{X}\left(\mathrm{E}_{X X}{ }_{x} \cdot \&_{x}\right.$ is a mathematician $)$

hence,

$\exists x\left(\bigvee_{x_{X}}={ }_{x} \cdot \&_{x}\right.$ is a mathematical problem $)$

was the "specifically isolated generic representation" of the argument

$\mathrm{A}_{\boldsymbol{x}}\left(\mathrm{x}\right.$ is a mathematician $\rightarrow V_{\mathrm{X}}(\mathrm{X}$ is a mathematical problem \&

$\sim_{x}$ is able to solve $\left.\mathrm{X}\right)$ )

$E_{\boldsymbol{X} \boldsymbol{X}}$ is a mathematician

hence,

$\mathrm{V} x \mathrm{x}$ is a mathematical problem.

And we could then state the Generic-representation Criterion this way:

A specific argument is formally valid if and only if its specifically isolated generic representation is formally valid

One might wonder whether McHeidegger would be willing to accept the Generic-representation Criterion as not only a true statement but as a complete and satisfactory account of the validity of specific inferences. If he were indeed willing to solve the problem of mixed inferences in that way, it would be interesting to hear his response to the questions posed at the end of the following rather lengthy speech:

If, as you say, the specific quantifiers are the fundamental quantifiers, the semantically primitive quantifiers, and if, as you say, the generic quantifiers are non-fundamental, semantically derived, and disjunctive, the criterion of formal validity for arguments couched in the quantifier-variable idiom amounts to this:

To determine whether an argument involving quantifiers is 
formally valid, replace each occurrence of a fundamental, semantically primitive quantifier in the argument with an occurrence of its non-fundamental, semantically derived, and disjunctive counterpart (restricted by the apposite placement of a suitable "logically inert" open sentence like ' $Z_{y}$ ' or ' $V x x$ $=x^{\prime}$ ); then test the resulting argument for validity by applying the rules set out in your favorite logic textbook to the occurrences of the non-fundamental, semantically derived, and disjunctive quantifiers it contains. If, therefore, one wishes to determine whether an argument involving quantifiers is formally valid, in the final analysis, the only inference-rules one will attend to are those that govern the non-fundamental, semantically derived, and disjunctive quantifiers. There is no need even to bother to formulate inference-rules that govern the fundamental, semantically primitive quantifiers, since, to test an argument for validity, one must first eliminate the fundamental, semantically primitive quantifiers; or at any rate one must permit the fundamental quantifiers to occur only in expressions like ' $E_{x} x=z$ ' and ' $V x x=y$ ' - expressions which have no more significance in the matter of determining inferential validity than (respectively) such significance as is contained in the descriptions "a sentence in which ' $z$ ' is free (and no other variable is free)" and "a sentence in which ' $y$ ' is free (and no other variable is free)".

Is that not a rather strange criterion of validity? Is it plausible to suppose that a linguistic item - in any syntactical category that is non-fundamental, semantically derived, and disjunctive plays an essential role in the criterion for determining whether an inference is formally valid? Is it plausible to suppose that quantifiers that are fundamental and semantically primitive have no role to play in the criterion for deciding the validity of arguments whose logical structures obviously depend on the way in which occurrences of quantifiers and of the variables they bind are distributed in their constituent sentences?

If McHeidegger refuses to regard the Generic-representation Criterion as the key to the solution of the problem of mixed inferences 
— if he rejects the Shallow Approach to this problem —, he will probably wish to propose an alternative solution. Might he propose the following solution?

The solution to the problem of mixed inferences is to "paraphrase away" all the non-fundamental and semantically derived quantifiers (that is, all the generic quantifiers) that occur in any mixed inference we propose to test for validity — systematically to rewrite the sentences in which they occur as sentences containing only fundamental and underived quantifiers. Consider first the particular quantifier. We eliminate occurrences of the particular quantifier by "disjunctive paraphrase" (working "from the inside out", as they say, starting with those generic quantifiers such that no generic quantifier occurs within their scope). For example, the sentence

$\exists x(x$ is a mathematical problem $\& \sim \exists y$ ( $y$ is a mathematician $\& y$ is able to solve $x)$ )

is paraphrased as

$E_{X}(x$ is a mathematical problem \&

$\sim[\mathrm{E} y(y$ is a mathematician $\& y$ is able to solve $x) \vee \vee y(y$ is a mathematician $\& y$ is able to solve $x)$ ]

v

VX $(\mathbf{X}$ is a mathematical problem \&

$\sim[\mathrm{Ey}$ ( $y$ is a mathematician \& $y$ is able to solve $\mathrm{x}) \vee \vee \mathrm{y}(\mathrm{y}$ is a mathematician $\& \mathrm{y}$ is able to solve $\mathrm{X})$ ].

(The paraphrase displays clearly the "disjunctive" character of the particular quantifier.)

Consider now the universal quantifier. (This example will be simpler than the previous example in that it will not involve a generic quantifier that occurs within the scope of a generic quantifier.) The sentence 
$\forall x$ ( $x$ is a problem in algebraic topology $\rightarrow x$ is a mathematical problem)

is paraphrased as

A $x$ ( $x$ is a problem in algebraic topology $\rightarrow x$ is a mathematical problem)

\section{\&}

A $\mathbf{x}(\mathrm{X}$ is a problem in algebraic topology $\rightarrow \mathrm{X}$ is a mathematical problem).

(Strictly speaking, it is only the particular quantifier that is in any sense — disjunctive. The universal quantifier, since it is the dual of the particular quantifier, is "conjunctive".) After all the generic quantifiers that occur in the constituent sentences of an argument have been eliminated by paraphrase, the resulting "purely specific" argument may be tested for validity by applying the rules of many-sorted logic.

But this proposal is unsatisfactory for a reason closely connected with our earlier observation that some "cross-modal generalizations" cannot be expressed without the use of the generic quantifiers. Consider the following cross-modal generalization:

$$
\forall x\left(\vee y y={ }_{x} \cdot v E_{y} y={ }_{x}\right)
$$

(Everything either subsists or exizts.) The method of paraphrase illustrated above yields, when applied to this sentence,

$$
\wedge \mathrm{x}\left(\mathrm{Vy} \mathrm{y}=\mathrm{x} \cdot \mathrm{vE}_{\mathrm{y}} \mathrm{y}=\mathrm{x}\right)
$$

$$
A_{x}\left(\vee y y=x \cdot v E_{y}=x\right) .
$$

Formally speaking, this sentence is simply a theorem of many-sorted logic. If the quantifiers have their intended meanings, it says (more or less) that everything subsistent is either subsistent or exiztent and everything exiztent is either subsistent or exiztent. The proposal is 
unsatisfactory because the paraphrases on which it relies reduce substantive meta-ontological theses to logical trivialities.

Here is a second solution to the problem of mixed inferences that a McHeideggerian might propose - and it is the only other proposal that occurs to me:

Use the Generic-representation Criterion, yes, but only to determine the validity of formal mixed inferences whose constituent sentences contain the generic quantifiers - the non-fundamental and semantically derived quantifiers. But to determine the validity of formal mixed inferences whose constituent sentences contain only fundamental and semantically primitive quantifiers, use the techniques of many-sorted logic.

As we have observed, however, an argument that contains specific quantifiers but no generic quantifiers is valid according to the rules of many-sorted logic if and only if it is valid according to the Generic-representation Criterion. (And, as we have noted, this is no deep result of mathematical logic.) This observation convinces me that a sentence containing specific quantifiers and sorted terms and its generic/unsorted "counterpart" are nothing more than notational variants. I will try to make this conviction of mine at least plausible (to anyone who does not already find it plausible) by means of a parable.

\section{6}

\section{McHeidegger Triumphant Confronts the Martians: A Parable}

By the end of the twenty-first century, McHeidegger and his disciples had triumphed. The McHeideggerian meta-ontology was taught in every university, and students of formal logic were taught manysorted logic. They were also taught unsorted or generic logic, but were strictly enjoined to use this logic only in very special cases namely, when engaged in reasoning from premises some of which could be expressed only by the use of the generic quantifiers.

One of the many shocks that followed the discovery in 2102 of the ancient hidden Martian civilization (the Martians dwelt in vast 
cities far beneath the surface of their planet) occurred when it transpired that the Martians were not McHeideggerians. The Martians were excellent logicians, and, in metaphysics, many of them were platonists. But they perversely insisted on employing only one particular/general quantifier-pair in all their reasoning - in their reasoning about every subject-matter and every kind of object. That is to say, their only quantifiers were the generic quantifiers. And, when they had learned Terrestrial languages and the human Principia-derived logical notation, they compounded their logical perversity by insisting that they could understand the so-called specific quantifiers only as restricted generic quantifiers. Indeed, when their logic texts began to incorporate material on Terrestrial logic, they went so far as to suggest that the specific quantification of Terrestrial logic was nothing more than a notational variant on appropriately restricted generic quantification. Their textbooks included what they were pleased to call translation algorithms, algorithms based on tables of schemata like this one:

$$
\begin{array}{ll}
\mathrm{E}_{x}(\ldots x \ldots) & \exists_{x}\left(\mathbf{Z}_{x} \&(\ldots x \ldots)\right) \\
\mathcal{A}_{x}(\ldots x \ldots) & \forall_{x}\left(\mathbf{Z}_{x} \rightarrow(\ldots x \ldots)\right) \\
\vee x(\ldots x \ldots) & \exists_{x}\left(\mathbf{S}_{x} \&(\ldots x \ldots)\right) \\
\wedge x(\ldots x \ldots) & \forall_{x}\left(\mathbf{S}_{x} \rightarrow(\ldots x \ldots)\right) .
\end{array}
$$

Martian students were actually taught to think of the schemata displayed in the left-hand column of this table as abbreviations of their counterparts in the right-hand column!

Such perversity led to much head-shaking among Terrestrial metaphysicians and logicians over the philosophical limitations of the Martian mind. Fortunately, however, it had no untoward practical consequences, since the Martians and the Terrestrials always agreed about which arguments expressed in the specific-quantifier notation were valid. (The Martians aggravated the effects of their perversity by pointing out that while they were able to get along without the specific quantifiers, Terrestrials were unable to get along without the generic quantifiers. They sometimes suggested that it was hard to see why the semantically fundamental quantifiers should be "option- 
al" and the derived quantifiers "required". That was just plain rude, when you think about it. And that was not the end of their rudeness, for it was not uncommon to hear Martian logicians say things like, "You Terrestrials at least see how we Martians claim to derive the specific quantifiers from the generic quantifiers - even if you insist that what we call a derivation is not properly so called. But could you just fill me in on how you Terrestrials derive the generic quantifiers from the specific quantifiers? - for I suppose that, by calling the generic quantifiers 'derived', you are claiming to have constructed such a derivation. I ask because I don't think I've ever seen the derivation actually written down anywhere". That was really rude.)

Here endeth the parable.

\section{7}

I have to say that I'm a Martian - by philosophical conviction if not by biological ancestry. And I have to say that I don't see what it could be that the Terrestrials (in the parable) know or are aware of or appreciate that the Martians don't know or aren't aware of or don't appreciate.

"Well, they don't know about, aren't aware of, and don't appreciate, the fact that there are two modes of being; and, further, they don't know (etc.) that existenz and subsistence are so different that each requires its own pair of specific quantifiers; and, further still, they don't know (etc.) that the generic quantifiers are not semantically fundamental but derived - semantically parasitic on the specific quantifiers, as it were."

To me, however, the fact that the Martians get along perfectly well without the specific quantifiers (as indeed Terrestrials did before the advent of McHeidegger), strongly suggests that there's just nothing to this idea of modes of being. (And let us not forget the fact that the Terrestrials, for their part, are not capable of getting along without the generic quantifiers.) It suggests to me that the so-called specific quantifiers are a manifestation of a fundamental meta-ontological error that I, a proponent of the "thin conception of being, have more than once contended is the foundation of the idea (endemic among the proponents of "thick" conceptions of being) that there are distinct and irreducible modes of being: the error of ascrib- 
ing to the being of a thing a feature that properly belongs to its nature. If I may quote myself (I was speaking of Sartre's distinction between être-pour-soi and être-en-soi, but the point I was making was a general one and applies to the position of any philosopher who postulates distinct modes of being):

There is, of course, a vast difference between free, conscious agents like ourselves and mere inanimate objects. I believe this quite as firmly as Sartre does. But to insist, as I do, that this difference does not consist in the one sort of thing's having a different sort of being from the other's is not to depreciate it. The vast difference between me and a table does not consist in our having vastly different sorts of being (Dasein, dass sein, "that it is"); it consists rather in our having vastly different sorts of nature (Wesen, was sein, "what it is"). If you prefer, what the table and I are like is vastly different. This is a perfectly trivial thing to say: that a vast difference between $\mathrm{A}$ and $\mathrm{B}$ must consist in a vast difference in their natures. But if a distinction can be made between a thing's being and its nature, this trivial truth is in competition with a certain statable falsehood. And if one denies the trivial at the outset of one's investigations, there is no hope for one later on. ${ }^{16}$

My choice of Sartre as an example of a philosopher whose philosophy exhibits the "fundamental meta-ontological error" whose character I am attempting to describe should not be taken to imply that I suppose that the error has been confined to Sartre's side of the English Chanel. That is by no means the case. Bertrand Russell, for example, has written, ${ }^{17}$

At the end of the preceding chapter, we saw that such entities as relations appear to have a being which is in some way different from that of physical objects, and also different from that of minds or sense-data. In the present chapter we have to consider what is the nature of this kind of being, and also what objects there are that have this kind of being.

We shall find it convenient only to speak of things existing when they are in space and time ... Thus thoughts and feelings, minds and physical objects exist. But universals do not exist in this sense; we shall say that they subsist or have being, ${ }^{18}$ where 'being' is opposed to 'existence' as being timeless.

16 "Being, Existence, and Ontological Commitment," in Metametaphysics: New Essays on the Foundations of Ontology (see n. 1), p. 477.

${ }^{17}$ The first quoted passage contains the opening words of Chapter IX of The Problems of Philosophy; the second occurs near the end of that chapter.

${ }^{18}$ Russell's 'existence'/'subsistence' distinction seems to be taken from Meinong; if so he has reproduced it imperfectly, particularly in the second passage. 
I have no real argument against the meta-ontological position presented in these two quotations. They do, however, tempt me to rant for a bit. I will allow myself to succumb to temptation. Here, in lieu of an argument, is my rant.

No, Russell, no! Relations are vastly different from tables, yes, but that's just to say that the members of one of those two classes of objects have vastly different natures from the members of the other - that the properties of relations are vastly different from the properties of tables. For example relations are, as you say, not in space and time and tables are in space and time. There. When you've said that, that's what you've said. Relations lack the property spatio-temporality and tables have it. That's an enormous difference between relations and tables, all right. (And of course, there are other things you might say: that things stand in relations and things don't - in that sense, at any rate - , stand in tables, or that relations exist necessarily and tables contingently; one could go on and on.) But when you've described the radically different properties that relations and tables have, you have not only done everything that is needed to describe the vast difference between relations and tables, you have done everything that can be done to describe it. That's what describing a vast difference is. Stop trying to do something more when there's nothing more to be done: stop trying to express the vastness of the difference between relations and tables by saying that they have different kinds of being. ${ }^{19}$

For Meinong, Existenz and Bestand are the two modes of Sein; he would certainly not have used 'Bestand' and 'Sein' interchangeably. And one does see his point: Would Russell really have been willing to say that Trinity College did not have being? He is truer to Meinong in the first passage, where he speaks of relations as having a different kind of being from "that of", e.g., physical objects.

${ }^{19}$ I thank Hannes Leitgeb and Chris Menzel for extremely helpful comments on a draft of this essay (comments that have led to extensive revisions). I also thank Daniel Durante for extensive correspondence on many of the matters raised in this paper, correspondence from which I learned a great deal, even though his arguments failed to convince me that his central thesis was correct - to wit, that it is wrong to suppose that there is such a thing as "the" existential or particular quantifier and such a thing as "the" universal quantifier. (His position is that "classical" logic has its own pair of quantifiers, and intuitionistic logic has its own pair of quantifiers - and so for paraconsistent logic, free logic, fuzzy logic, and many other "logics." Despite his very able defense of this thesis, I remain unconvinced.) Finally, I wish to thank Kris McDaniel for his insightful comments on an earlier 
Peter van Inwagen

Department of Philosophy The University of Notre Dame Notre Dame, Indiana 46556-4619 574-631-5910 dept/ 574-277-7427 home Fax: 574-631-8209 vaninwagen.1@nd.edu

\section{References}

Enderton, Herbert B. 2001. A Mathematical Introduction to Logic, 2nd Edn. Harcourt: Academic Press.

Inwagen, Peter van. 2009. Being, Existence, and Ontological Commitment. In Metametaphysics: New Essays on the Foundations of Ontology. Edited by David Chalmers, David Manley and Ryan Wasserman. Oxford: Oxford University Press.

McDaniel, Kris. 2009. Wavs of Being. In Metametaphysics: New Essays on the Foundations of Ontology. Edited by David Chalmers, David Manley and Ryan Wasserman. Oxford: Oxford University Press.

Russell, Bertrand. 1912. The Problems of Philosophy.

version of this paper. I am sorry to say that, insightful as they were, they did not convince me that there is any flaw in the case I have presented against the idea of modes of being. 